\title{
Dimensionado de las escaleras protegidas en caso de incendio: un reto para los métodos prescriptivos (El modelo del CTE)
}

\section{Dimensioning of the protected stairs in case of fire: a challenge for prescriptive methods (The CTE model)}

\author{
J. B. Echeverría ${ }^{(*)}$, M. Fernández-Vigil $^{(*)}$, B. Gil ${ }^{(*)}$
}

\section{RESUMEN}

El dimensionado de las escaleras en caso de incendio se encuentra entre las características más importantes del diseño arquitectónico actual. La mayor complejidad de los edificios (usos mixtos, espacios con alta ocupación, personas con discapacidad, edificios en altura, etc.) se suma a nuevos hábitos sociales, y las actividades que se desarrollaban tradicionalmente en las partes bajas de los edificios, tienden a ocupar los pisos más altos. Este artículo muestra que los cálculos prescriptivos para el dimensionado de las escaleras protegidas son incapaces de enfrentarse a este reto por estar basados en modelos simplificados, solamente válidos para edificios y distribuciones convencionales. Analiza el caso del Código Técnico de la Edificación español, mostrando su debilidad, e introduce los conceptos de diseño prestacional necesarios para entender el problema. Concluye que cualquier diseño complejo debe abordarse estudiando el movimiento real de los ocupantes, que puede ser modelizado con la ayuda de herramientas computacionales.

Palabras clave: escaleras protegidas, dimensionado, capacidad, densidad, flujo.

\section{ABSTRACT}

Dimensioning the stairs in case of fire is amongst the most important features in today architecture design. A bigger complexity of the buildings (mixed uses, crowded spaces, occupants with disabilities, tall structures, etc.) is added to new social habits, and the activities traditionally placed on the lowest parts of the buildings tend to occupy the upper floors. This article shows that the prescriptive calculations for the dimensioning of protected stairs are unable to face this challenge because they are based on simplified models, only valid for conventional buildings and distributions. It analizes the Spanish Technical Building Code, showing its weakness, and introduces the necessary performance-based design concepts to understand the problem. It concludes that any complex design must be addressed studying the real movement of the occupants, that can be modelled with the use of computational tools.

Keywords: Protected stairs, sizing, capacity, density, flow.

(*) Universidad de Navarra

Persona de contacto/Corresponding author: mfernandez-@alumni.unav.es (M. Fernández-Vigil)

ORCID: http://orcid.org/oooo-0002-4438-6449 (J. B. Echeverría); http://orcid.org/oooo-0002-4309-1606

(M. Fernández-Vigil); http://orcid.org/oooo-0002-2348-0487 (B. Gil)

Cómo citar este artículo/Citation: J. B. Echeverría, M. Fernández-Vigil, B. Gil. (2018). Dimensionado de las escaleras protegidas en caso de incendio: un reto para los métodos prescriptivos (El modelo del CTE). Informes de la Construcción, 70(550): e258. https://doi. org/10.3989/id.59396

Copyright: (c) 2018 CSIC. Este es un artículo de acceso abierto distribuido bajo los términos de la licencia de uso y distribución Creative Commons Reconocimiento 4.o Internacional (CC BY 4.0). 


\section{INTRODUCCIÓN}

Los códigos de edificación ofrecen, en la mayoría de los casos, soluciones aceptables de carácter prescriptivo para el diseño y cálculo de los diferentes elementos necesarios para el cumplimiento de las exigencias contempladas por la legislación. Estas soluciones son perfectamente válidas, y así son aceptadas, tanto por los diseñadores como por las diferentes administraciones encargadas de su control. Están basadas en la experiencia y se elaboran con la mejor información de la que se dispone en el momento en el que son redactadas. Su carácter prescriptivo y su aceptación incuestionable hacen que sean, además, las soluciones más utilizadas. Este hecho, sin embargo, oculta con frecuencia las bases y los modelos a partir de los cuales estas soluciones se han desarrollado, adquiriendo una apariencia casi mágica (1).

Esta problemática, de carácter general, se hace muy evidente en el caso de la Seguridad en Caso de Incendio, en la que un complejísimo problema que relaciona un diseño arquitectónico, la iniciación y propagación de un fuego y la conducta incierta de unos ocupantes se aborda con medidas prescriptivas como son el tamaño máximo y disposición de los sectores, los recorridos máximos hasta las salidas o sencillas fórmulas para el cálculo de los elementos de evacuación.

Este artículo ejemplifica esta problemática en el caso del método prescriptivo propuesto para el dimensionado de las escaleras protegidas en el Código Técnico de la Edificación (CTE), que ha sido arrastrado a la actual legislación española desde normativas anteriores. Analiza el modelo excesivamente simple de evacuación en el que está basado y pone en evidencia sus limitaciones en situaciones de ocupaciones complejas, cada vez más habituales. Se utilizan para ello conceptos manejados por la ingeniería de protección contra incendios, a nivel internacional, en el diseño prestacional de los elementos de evacuación, que están camuflados tras las sencillas expresiones matemáticas que ofrece el CTE. Se proponen soluciones de diseño para que se pueda coordinar el flujo proveniente de una planta determinada con ocupación sensiblemente superior a la del resto con el de la escalera. Se utilizan para ello tanto sencillos cálculos manuales como simulaciones con Pathfinder.

Los objetivos últimos son tres. El primero de ellos, y más importante, es alertar sobre el riesgo que pueden conllevar determinados diseños de escaleras protegidas amparados tras un método prescriptivo totalmente aceptado por la legislación española. El segundo es recomendar que cualquier problema complejo de evacuación sea analizado utilizando criterios y métodos prestacionales como los aquí utilizados.
El tercero es promover una cultura del análisis de lo que se esconde tras las soluciones aceptables, dinamizando la relación entre legisladores, investigadores y diseñadores.

\section{LA SEGURIDAD EN CASO DE INCENDIO Y LA EVACUACIÓN DE OCUPANTES EN EL CTE}

El Real Decreto 314/2006 de aprobación del CTE (2), mostraba su alineación con el enfoque basado en prestaciones (3)(4) propugnado por las principales Organizaciones Internacionales relacionadas con códigos de la edificación, como el Consejo Internacional de la Edificación o el Comité Interjurisdiccional de Colaboración Reglamentaria, concluyendo que la normativa debía promover la investigación y no dificultar el progreso tecnológico.

De forma coherente con ese enfoque, el CTE se divide en dos partes. La primera contiene las disposiciones y condiciones generales de aplicación y las exigencias básicas que deben cumplir los edificios y la segunda, los denominados Documentos Básicos (DB) que garantizan el cumplimiento de las exigencias básicas contenidas en la parte primera.

Para la Seguridad en Caso de Incendio, el CTE desarrolla seis exigencias básicas que, básicamente, se corresponden con los requisitos básicos enumerados en la Directiva de Productos de Construcción (5). La Tabla 1 muestra la correspondencia entre ambos documentos, ilustrando sobre el origen de nuestra actual legislación de edificación.

El Documento Básico DB-SI especifica parámetros objetivos y procedimientos cuyo cumplimiento asegura la satisfacción de las exigencias básicas y la superación de los niveles mínimos de calidad propios del requisito básico de seguridad en caso de incendio. Su contenido deriva en gran medida de las anteriores Normas Básicas de la Edificación, NBE CPI 81 (6) NBE CPI 91 (7) y NBE CPI 96 (8), que eran puramente prescriptivas. Ese es el caso del cálculo de los elementos de evacuación.

Como la Exigencia Básica Evacuación de Ocupantes establece que "El edificio dispondrá de los medios de evacuación adecuados para que los ocupantes puedan abandonarlo o alcanzar un lugar seguro dentro del mismo en condiciones de seguridad" (9), el dimensionado de los elementos de evacuación mediante el uso del Documento Básico debería garantizar el cumplimiento de la Exigencia Básica Evacuación de Ocupantes y el cumplimiento del Objetivo del Requisito Básico de Seguridad en caso de Incendio. $Y$ ese es el caso de edificios sencillos y de poca altura. Ese dimensionado, no obstante, podría subestimar importantes riesgos en el caso de edificios complejos y con grandes densidades de ocupación

Tabla 1. Relación entre los requisitos básicos de Seguridad en caso de incendio en la Directiva de Productos de Construcción y en el CTE.

\begin{tabular}{|l|l|}
\hline $\begin{array}{l}\text { REGULATION (EU) No 3O5/2011 OF THE EUROPEAN PARLIA- } \\
\text { MENT AND OF THE COUNCIL of 9 March } 2011\end{array}$ & CÓDIGO TÉCNICO \\
\hline $\begin{array}{l}\text { (a) the load-bearing capacity of the construction can be assumed for a } \\
\text { specific period of time }\end{array}$ & (SI-6) Resistencia de la Estructura \\
\hline $\begin{array}{l}\text { (b) the generation and spread of fire and smoke within the construction } \\
\text { works are limited }\end{array}$ & (SI-1) Propagación Interior \\
\hline $\begin{array}{l}\text { (c) the spread of fire to neighbouring construction work is limited } \\
\text { (d) occupants can leave the construction works or be rescued by other } \\
\text { means }\end{array}$ & (SI-2) Propagación Exterior \\
\hline (e) the safety of rescue teams is taken into consideration & (SI-3) Evacuación de ocupantes \\
\hline & (SI-5) Intervención de los bomberos \\
\hline
\end{tabular}




\begin{tabular}{|c|l|c|l|}
\hline \multicolumn{3}{|c|}{ Nomenclatura } \\
\hline $\mathrm{A}$ & Anchura del elemento de evacuación $(\mathrm{m})$ & $\mathrm{S}$ & Superficie de la escalera en el conjunto de las plantas (m2) \\
$\mathrm{Fe}$ & Flujo específico en la escalera $(\mathrm{p} / \mathrm{s} / \mathrm{m})$ & $\mathrm{Se}$ & Superficie de la escalera en cada planta (m2) \\
$\mathrm{Fi}$ & Flujo específico en la planta $\mathrm{i}(\mathrm{p} / \mathrm{s} / \mathrm{m})$ & $\mathrm{t}$ & Tiempo (s) \\
$\mathrm{i}$ & $\mathrm{i}^{\mathrm{a}}$ planta & te & Tiempo en la escalera (s) \\
$\mathrm{n}$ & Número de plantas servido por la escalera & t paso & Tiempo de paso (s) \\
$\mathrm{P}$ & Número de personas asignado a la escalera en el conjunto de & t conf & Tiempo de confluencia (s) \\
& las plantas & t esp & Tiempo de espera (s) \\
$\mathrm{Pte}$ & Número de personas total en el interior de la escalera & & \\
$\mathrm{Pe}$ & Número de personas en la escalera entre dos plantas conse- & & \\
$\mathrm{Pi}$ & cutivas & & \\
\hline
\end{tabular}

\section{EL CÁlCULO DE LOS ELEMENTOS DE EVACUACIÓN EN EL CTE}

El cálculo de los elementos de evacuación en el DB-SI3 del CTE es muy simple. Lo único que tiene en cuenta es la relación entre el número de personas asignado al elemento de evacuación (P) y la anchura de dicho elemento (A). No se vincula, aparentemente, al movimiento real de los ocupantes, ni al tiempo requerido para ese movimiento.

Las expresiones utilizadas responden a dos modelos:

1. Para puertas, pasillos y escaleras no protegidas, la anchura de los elementos es solamente función del número de personas asignado al elemento de evacuación. Aunque no se expresa directamente, esto corresponde a un flujo a través del elemento considerado.

2. Para pasillos y escaleras protegidos, la anchura de los elementos es función del número de personas asignado al elemento de evacuación y de la superficie del elemento (S). Esto implica que, además del flujo, se considera la capacidad del elemento para contener a los ocupantes, a razón de 3 personas por metro cuadrado. En consecuencia, la capacidad del elemento aumenta cuando la altura del edifico aumenta, en el caso de las escaleras y cuando la longitud del pasillo aumenta, en el caso de los pasillos.

La Tabla 2 recoge la anchura de los elementos de evacuación considerados en el DB-SI3.

Una característica importante del diseño de estos elementos de evacuación, es que pueden acumularse a lo largo de un mismo recorrido de evacuación. Por ejemplo, un ocupante podría atravesar primero un pasillo y luego una puerta intermedia para, finalmente, llegar al final del recorrido computable, que podría ser otra puerta. Los tres elementos estarían separados y, el hipotético tiempo total de recorrido, incluiría los pasos a través de esos elementos considerados, pero también el tiempo de desplazamiento entre ellos.

\section{EL CÁlCULO DE LAS ESCALERAS PROTEGIDAS EN EL DB-SI3 DEL CTE}

Una escalera protegida, de acuerdo con la definición contenida en la Terminología del DB-SI3, constituyen una salida de planta. Esto quiere decir que una vez alcanzada la puerta de dicha escalera, la longitud del recorrido posterior no computa a efectos del cumplimiento de los límites de evacuación. Es una escalera de trazado continuo hasta su desembarco en la planta de salida del edificio que, en caso de incendio, constituye un recinto suficientemente seguro para permitir que los ocupantes puedan permanecer en el mismo durante un determinado tiempo. Es un recinto destinado exclusivamente a circulación, está compartimentado del resto del edificio mediante elementos EI 120 y dispone de un sistema de protección frente al humo.

El estudio de la anchura de las escaleras en los códigos de edificación ha sido objeto de investigación durante muchos años (10)(11). Como ya se ha visto, el cálculo de las escaleras protegidas queda definido en el DB SI-3 por la expresión:

$$
\mathrm{PE} \leq 3 \mathrm{~S}+160 \mathrm{As}
$$

El modelo propuesto responde a un método capacitivo, inicialmente propuesto en 1952 en Gran Bretaña (12). Éste, considera que el flujo de personas desde todas las plantas hacia una escalera protegida se produce en dos etapas:

1. El llenado de cada tramo entre plantas de la escalera desde la planta inmediatamente superior. En este tiempo un flujo ininterrumpido tiene acceso a la escalera desde las diferentes plantas.

Tabla 2. Anchura de elementos de evacuación, de acuerdo con DB SI-3.

\begin{tabular}{|c|c|c|}
\hline Elemento & \multicolumn{2}{|c|}{ Anchura (m) } \\
\hline Puertas, pasillos y ecaleras & \multicolumn{2}{|l|}{$\mathrm{A} \geq \mathrm{P} / 200$} \\
\hline \multirow{2}{*}{ Escaleras no protegidas } & Descendente & $\mathrm{A} \geq \mathrm{P} / 16 \mathrm{o}$ \\
\hline & Ascendente & $\mathrm{A} \geq \mathrm{P} /(160-10 h)$ \\
\hline Pasillos protegidos & \multicolumn{2}{|l|}{$\mathrm{P} \leq 3 \mathrm{~S}+200 \mathrm{~A}$} \\
\hline Escaleras protegidas (Descendente y Ascendente) & \multicolumn{2}{|l|}{$\mathrm{E} \leq 3 \mathrm{~S}+160 \mathrm{As}$} \\
\hline \multicolumn{3}{|c|}{$\begin{array}{l}\text { A = Anchura (m) } \\
\text { As = Anchura de la escalera protegida en su desembarco en la planta del edificio } \\
\mathrm{P} \text { = Numero de ocupantes asignados al elemento de evacuación } \\
\mathrm{h} \text { = Altura en evacuación ascendente } \\
\mathrm{S} \text { = Superficie de la escalera } \\
\mathrm{E} \text { = Suma de los ocupantes asignados a la escalera en la planta considerada, más los de las plantas situadas por debajo o } \\
\text { por encima de ella hasta la planta de salida del edificio (según se trate de una escalera ascendente o descendente, respec- } \\
\text { tivamente). }\end{array}$} \\
\hline
\end{tabular}


2. El flujo posterior tras el llenado, que modifica el inicial y queda condicionado por el de salida en la planta de desembarco de la escalera. A partir de ese momento el flujo de personas que pueden entrar en la escalera en sus diferentes plantas, se equilibra con el de salida.

La expresión [1] define las asunciones del modelo considerado por el legislador:

Asunción 1. El flujo de entrada en la escalera, antes de llenarse, es continuo y uniforme, y está condicionado por la anchura de la escalera en cada planta.

Asunción 2. La escalera puede contener 3 personas por m2, tanto en rellanos como en tramos.

Asunción 3. El flujo a través de la escalera, una vez llena y con las características de ocupación establecidas, es continuo y uniforme y está definido por 160As

Asunción 4. Las puertas de acceso a la escalera en las diferentes plantas y de salida en la planta de descarga de la misma, no condicionan los flujos considerados en la escalera, ya que sus anchuras son, al menos, las necesarias para mantenerlos.

Asunción 5.. En la planta de descarga de la escalera no hay ocupantes que acceden a la misma, ni obstáculos que impiden que el flujo se mantenga.

Hay dos observaciones iniciales que pueden hacerse a la vista de [1]:

1. La primera es que el número $\mathrm{P}$ de personas que puede asignarse a la escalera, es mayor cuánto más alta es la escalera. Al aumentar el número de plantas, n, aumenta $\mathrm{S}$, ya que $\mathrm{S}=\mathrm{nSe}$ en el caso de tramos de escalera iguales y $\mathrm{S}=\sum$ Sei en el caso de tramos de escalera diferentes. Esto es así, aunque la anchura, A, se mantenga.

2. La segunda es que, al ser As la anchura de la escalera en su desembarco en la planta de salida del edificio, hay una indefinición sobre la anchura de la escalera en tramos superiores. La anchura As, como ya se ha visto, controla el flujo de paso en la planta de desembarco, pero no informa sobre otros flujos escaleras arriba que podrían variar si variara la anchura. Por esta razón, y por otras de pura economía espacial, las escaleras protegidas se suelen dimensionar con una anchura constante a lo largo de todo su recorrido.

Como otros códigos prescriptivos, el Documento Básico SI-3, ofrece una tabla para el cálculo directo de escaleras de trazado sencillo (Tabla 3). Esta tabla es habitualmente utilizada por los diseñadores, ya que representa una escalera optimizada, con la mayor anchura posible en la menor superficie de planta.

La Tabla 3 considera que la anchura de la escalera es igual en todo el desarrollo de la misma, por lo que consideraremos:

$$
\mathrm{PE} \leq 3 \mathrm{~S}+16 \mathrm{OA}
$$

En la Figura 1, se representa una escalera protegida tipo de acuerdo con las condiciones de la Tabla3. Para ello se han calculado las dimensiones resultantes en función de la Anchura, A, y de la Superficie de la escalera en una planta, Se, que se obtiene en función de los ocupantes que puede albergar la escalera en cada planta, $\mathrm{Pe} / 3$.

Para ello se ha seguido el criterio lógico de que el descansillo mantiene la anchura de los tramos y que la superficie en exceso quede del lado de la apertura de la puerta en cada planta. Se ha considerado una altura entre plantas de $3,5 \mathrm{~m}$, salvada por dos tramos de 10 peldaños cada uno (huella de $30 \mathrm{~cm}$. y contrahuella de $17,5 \mathrm{~cm}$.). Además, se ha considerado un hueco de $10 \mathrm{~cm}$ entre las zancas, para permitir la integración de las barandillas. La Tabla 4 recoge la geometría de esta escalera para las diferentes anchuras de la Tabla 3.

La última columna de la Tabla 4 recoge la relación entre la superficie de la escalera en cada planta y la anchura de la misma, que crece al aumentar la anchura.

Tabla 3. Tabla 4. 5 DB SI, CTE.

\begin{tabular}{|c|c|c|c|c|c|c|c|c|}
\hline \multicolumn{9}{|c|}{ Capacidad de evacuación de las escaleras en función de su anchura } \\
\hline \multirow{3}{*}{$\begin{array}{l}\text { Anchura de la } \\
\text { escalera en m }\end{array}$} & \multirow{2}{*}{\multicolumn{2}{|c|}{ Escalera no protegida }} & \multirow{2}{*}{\multicolumn{6}{|c|}{$\begin{array}{c}\text { Escalera protegida (evacuación descendente o ascendente } \\
\text { No de plantas }^{\circ} \text {. }\end{array}$}} \\
\hline & & & & & & & & \\
\hline & $\begin{array}{l}\text { Evacuación } \\
\text { descendente }\end{array}$ & $\begin{array}{l}\text { Evacuación } \\
\text { descendente }\end{array}$ & 2 & 4 & 6 & 8 & 10 & Cada planta más \\
\hline 1,00 & 132 & 160 & 224 & 288 & 352 & 416 & 480 & +32 \\
\hline 1,10 & 145 & 176 & 248 & 320 & 392 & 464 & 536 & +36 \\
\hline 1,20 & 158 & 192 & 274 & 356 & 438 & 520 & 602 & +41 \\
\hline 1,30 & 171 & 208 & 302 & 396 & 490 & 584 & 678 & +47 \\
\hline 1,40 & 184 & 224 & 328 & 432 & 536 & 640 & 744 & +52 \\
\hline 1,50 & 198 & 240 & 356 & 472 & 588 & 704 & 820 & +58 \\
\hline 1,60 & 211 & 256 & 384 & 512 & 640 & 768 & 896 & +64 \\
\hline 1,70 & 224 & 272 & 414 & 556 & 698 & 840 & 982 & +71 \\
\hline 1,80 & 237 & 288 & 442 & 596 & 750 & 904 & 1058 & +77 \\
\hline 1,90 & 250 & 304 & 472 & 640 & 808 & 976 & 1144 & +84 \\
\hline 2,00 & 264 & 320 & 504 & 688 & 872 & 1056 & 1240 & +92 \\
\hline 2,10 & 277 & 336 & 534 & 732 & 930 & 1128 & 1326 & +99 \\
\hline 2,10 & 290 & 352 & 566 & 780 & 994 & 1208 & 1422 & $\begin{array}{l}+107 \\
+107\end{array}$ \\
\hline 2,30 & 303 & 368 & 598 & 828 & 1058 & 1288 & 1518 & +115 \\
\hline 2,40 & 316 & 384 & 630 & 876 & 1122 & 1368 & 1614 & +123 \\
\hline \multicolumn{9}{|c|}{ Número de ocupantes que pueden utilizar la escalera } \\
\hline
\end{tabular}




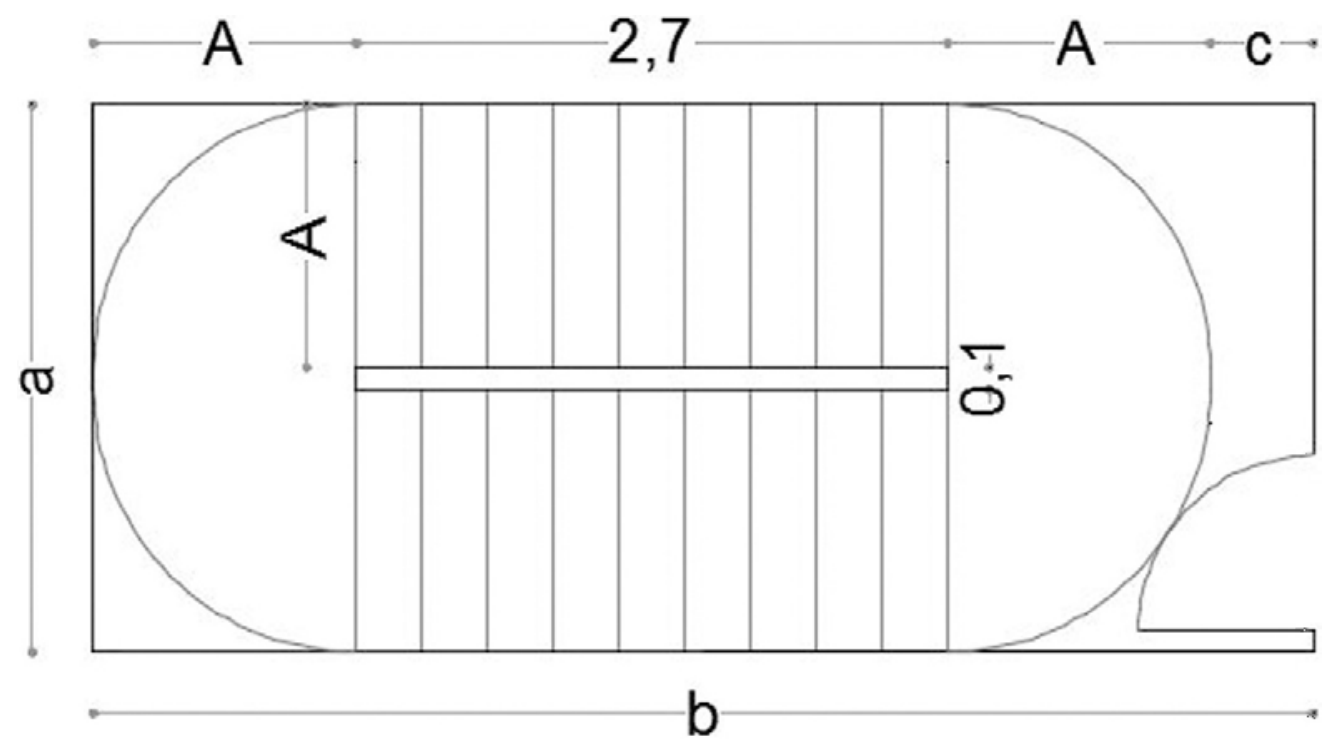

Figura 1. Diseño de Escalera protegida considerado de acuerdo con Tabla 3.

Tabla 4. Geometría de las escaleras consideradas en la Tabla 3.

\begin{tabular}{|c|c|c|c|c|c|c|c|}
\hline $\begin{array}{c}\text { Anchura de la } \\
\text { escalera en m } \\
\mathbf{A}\end{array}$ & $\mathbf{P e}$ & $\begin{array}{c}\mathbf{a} \\
\mathbf{m}\end{array}$ & $\begin{array}{c}\mathbf{b} \\
\mathbf{m}\end{array}$ & $\begin{array}{c}\mathbf{c} \\
\mathbf{m}\end{array}$ & $\begin{array}{c}\mathbf{a} \mathbf{x} \mathbf{b} \\
\mathbf{m} 2\end{array}$ & $\begin{array}{c}\text { Se } \\
\mathbf{m 2}\end{array}$ & $\mathbf{S e} / \mathbf{A}$ \\
\hline 1,00 & 32 & 2,1 & 5,21 & 0,51 & 10,94 & 10,67 & 10,67 \\
\hline 1,10 & 36 & 2,3 & 5,33 & 0,43 & 12,27 & 12,00 & 10,91 \\
\hline 1,20 & 41 & 2,5 & 5,57 & 0,47 & 13,94 & 13,67 & 11,39 \\
\hline 1,30 & 47 & 2,7 & 5,90 & 0,60 & 15,94 & 15,67 & 12,05 \\
\hline 1,40 & 52 & 2,9 & 6,07 & 0,57 & 17,60 & 17,33 & 12,38 \\
\hline 1,50 & 58 & 3,1 & 6,32 & 0,62 & 19,60 & 19,33 & 12,89 \\
\hline 1,60 & 64 & 3,3 & 6,55 & 0,65 & 21,60 & 21,33 & 13,33 \\
\hline 1,70 & 71 & 3,5 & 6,84 & 0,74 & 23,94 & 23,67 & 13,92 \\
\hline 1,80 & 77 & 3,7 & 7,01 & 0,71 & 25,94 & 25,67 & 14,26 \\
\hline 1,90 & 84 & 3,9 & 7,25 & 0,75 & 28,27 & 28,00 & 14,74 \\
\hline 2,00 & 92 & 4,1 & 7,55 & 0,85 & 30,94 & 30,67 & 15,33 \\
\hline 2,10 & 99 & 4,3 & 7,74 & 0,84 & 33,27 & 33,00 & 15,71 \\
\hline 2,10 & 107 & 4,5 & 7,99 & 0,89 & 35,94 & 35,67 & 16,21 \\
\hline 2,30 & 115 & 4,7 & 8,21 & 0,91 & 38,60 & 38,33 & 16,67 \\
\hline 2,40 & 123 & 4,9 & 8,42 & 0,92 & 41,27 & 41 & 17,08 \\
\hline
\end{tabular}

Esta relación (Fig. 2), viene dada por la expresión:

$$
\mathrm{Se}=10,374 \mathrm{~A}^{\wedge} 1,5581
$$

Sustituyendo en [1] podemos obtener el número de personas que pueden utilizar la escalera en función del número de plantas y la anchura [4]

$$
\mathrm{E} \leq 3 \mathrm{n}\left(10,374 \mathrm{~A}^{\wedge} 1,5581\right)+160 \mathrm{~A}
$$

Aunque el Documento Básico SI-3 no se refiere a las condiciones de movimiento de los ocupantes que soportan este modelo, sabemos que indudablemente el legislador las ha debido tener en cuenta. Las ya mencionadas NBE-CPI 91 y NBE-CPI 96, que incluían las mismas fórmulas y la Tabla 3 en su articulado, sí especificaban que las fórmulas estaban establecidas bajo la hipótesis de que todos los ocupantes pueden traspasar una salida en un tiempo máximo de 2,5 minutos (150 segundos).

La consideración de que si un espacio es evacuado en menos de 2,5 se disminuye considerablemente el riesgo de que los ocupantes puedan sufrir daños derivados de un incendio fue incorporada a numerosos códigos de edificación tras el incendio del Empire Palace Theater en Edimburgo, en 1911 (13).

\section{CONCEPTOS PRESTACIONALES BÁSICOS EN EL MOVIMIENTO DE EVACUACIÓN}

Predecir el movimiento de los ocupantes durante la evacuación es un aspecto esencial del análisis prestacional de la seguridad en caso de incendio en edificios, pero una tarea difícil. Como ya se ha comentado, los ocupantes se desplazan en el edificio horizontalmente, atravesando pasos y puertas, y verticalmente, utilizando escaleras. La utilización de elementos mecánicos, tales como escaleras mecánicas y ascensores, no es considerada válida por la legislación española y, aunque esto podría ser cuestionable en algunos casos, no es objeto del presente artículo.

Lo que caracteriza la evacuación en caso de incendio es que ésta se realiza en paralelo al desarrollo de un incendio que la puede condicionar muy seriamente. Se considera que si el 


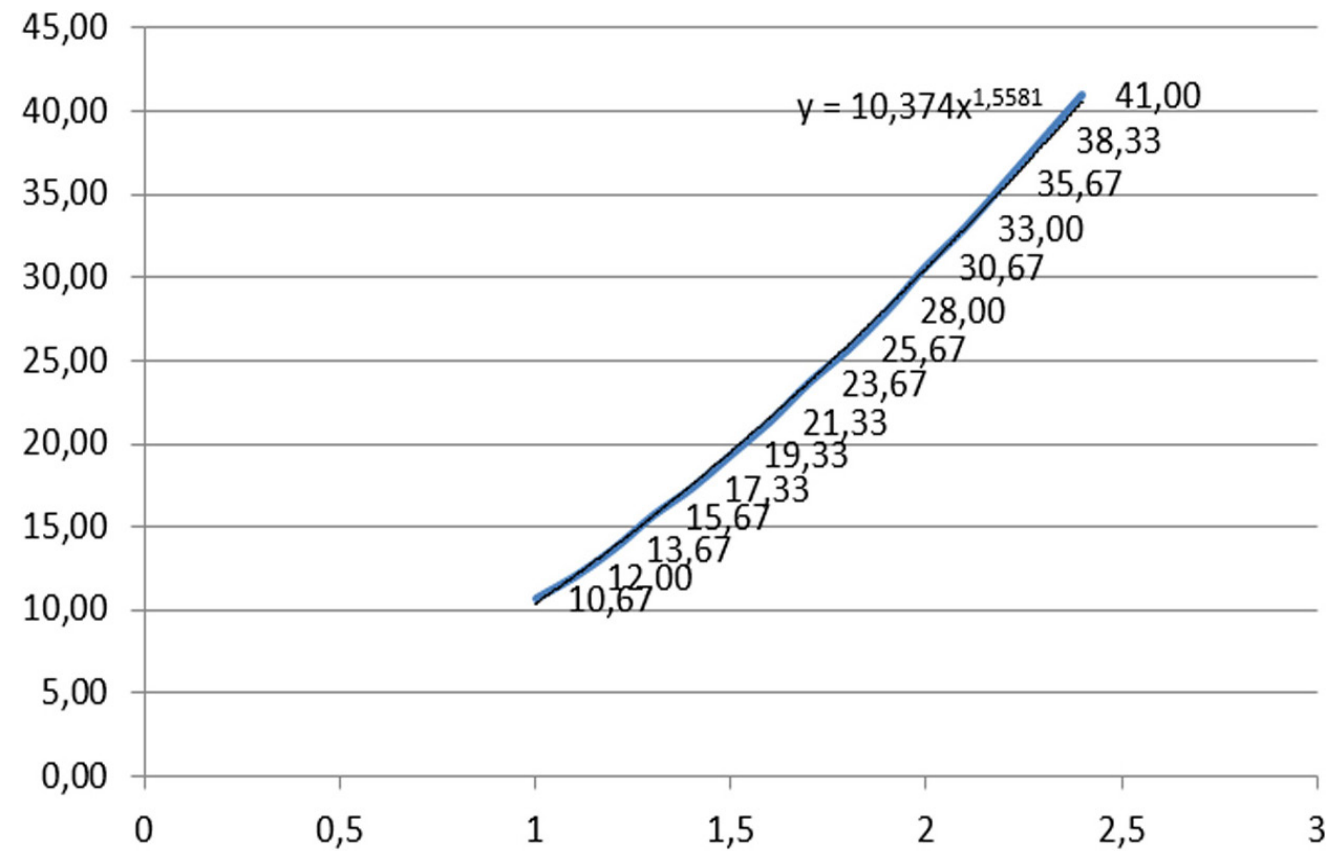

Figura 2. Relación entre el Ancho de la escalera (A) y la Superficie de la escalera en cada planta (Se).

tiempo disponible para la evacuación en condiciones de seguridad, ASET (Available Safe Escape Time) es mayor que el tiempo requerido para la evacuación en condiciones de seguridad, RSET (Required Safe Escape Time) más un margen de seguridad, se satisface la Exigencia Básica de evacuación de ocupantes (14). La relación entre ambos se encuentra descrita en la ISO/TR 16738:2009 (15).

Los factores fundamentales que afectan al ASET son la tasa de crecimiento del incendio y el tiempo en el que el recinto considerado se llena de humo, ya que se asume que la evacuación se produce por debajo del nivel de la capa de humo (16).

El RSET se obtiene cuando todos los ocupantes alcanzan un lugar seguro y de acuerdo con la terminología simplificada descrita en el Anexo H de la ISO/TR 16738:2009, se pueden formular dos hipótesis

1. Densidad de ocupación baja, sin que se produzcan colas en los cuellos de botella

$$
\text { RSET } 1=\text { tdet }+ \text { ta }+ \text { tpre }+ \text { ttra(pres })
$$

2. Densidad de ocupación elevada, con colas en los cuellos de botella

$$
\mathrm{RSET} 2=\mathrm{tdet}+\mathrm{ta}+\text { tpre }+ \text { ttra(pres })+\mathrm{ttra}(\text { queue })
$$

donde:

t det es el tiempo de detección

t a es el tiempo de alarma

t pre es el tiempo de pre-movimiento, desde la señal de alarma hasta que el primer ocupante se pone en movimiento

t tra (pres) es el tiempo para que el ocupante en condiciones más desfavorable llegue hasta una salida que conduzca a un lugar seguro t tra (queue) es el máximo tiempo de cola en la salida

La diferencia fundamental entre ambas hipótesis es que, si la densidad de ocupación es muy baja, los ocupantes pueden atravesar el elemento de la salida sin detenerse y, por tanto, sin crear tapones. Esta diferencia, ya avanza la influencia que en la velocidad de evacuación tiene la densidad, definida como la medida del grado de ocupación en una ruta de evacuación y expresada en personas por unidad de área. Nelson y Mowrer (17) establecieron las relaciones entre las velocidades y la densidad, tanto en desplazamientos horizontales como en escaleras.

El Flujo específico Fs (P/s/m), se define como el flujo de personas que atraviesa un punto en una ruta de evacuación por unidad de tiempo y por unidad de ancho efectivo, A. Es el producto de la Densidad (P/m2) y la Velocidad $(\mathrm{m} / \mathrm{s})$ y se expresa en personas $/ \mathrm{s} / \mathrm{m}$

$$
\mathrm{Fs}=\mathrm{D}^{*} \mathrm{~V}
$$

La información que utilizamos para el flujo de ocupantes, se avanzó en el trabajo de Fruin (18) (19), Pauls (20), Predtechenskii y Milinskii (21). La Fig. 3, del Handbook de la SFPE (22) muestra la relación entre el Flujo Específico y la densidad. En ella se observa que los valores máximos de Flujo Específico en movimiento horizontal (pasillos, rampas o puertas), es de aproximadamente $1,3 \mathrm{p} / \mathrm{s} / \mathrm{m}$ y en escaleras, dependiendo de las dimensiones de huellas y contrahuellas, se sitúa entre o,85 $\mathrm{p} / \mathrm{s} / \mathrm{m}$ y $1,10 \mathrm{p} / \mathrm{s} / \mathrm{m}$. En ambos casos los Flujos máximos se corresponden con densidades de unos $1,8 \mathrm{p} / \mathrm{m} 2$.

\section{ANÁLISIS PRESTACIONAL DEL MÉTODO EMPLEADO PARA EL CÁLCULO DE LAS ESCALERAS PROTEGIDAS EN EL CTE}

Ante la falta de información disponible en el DB-SI3 se procede a realizar un análisis inverso para intentar alumbrar las bases que sustentan el modelo elegido por el legislador para representar las condiciones de la evacuación en las escaleras protegidas. 


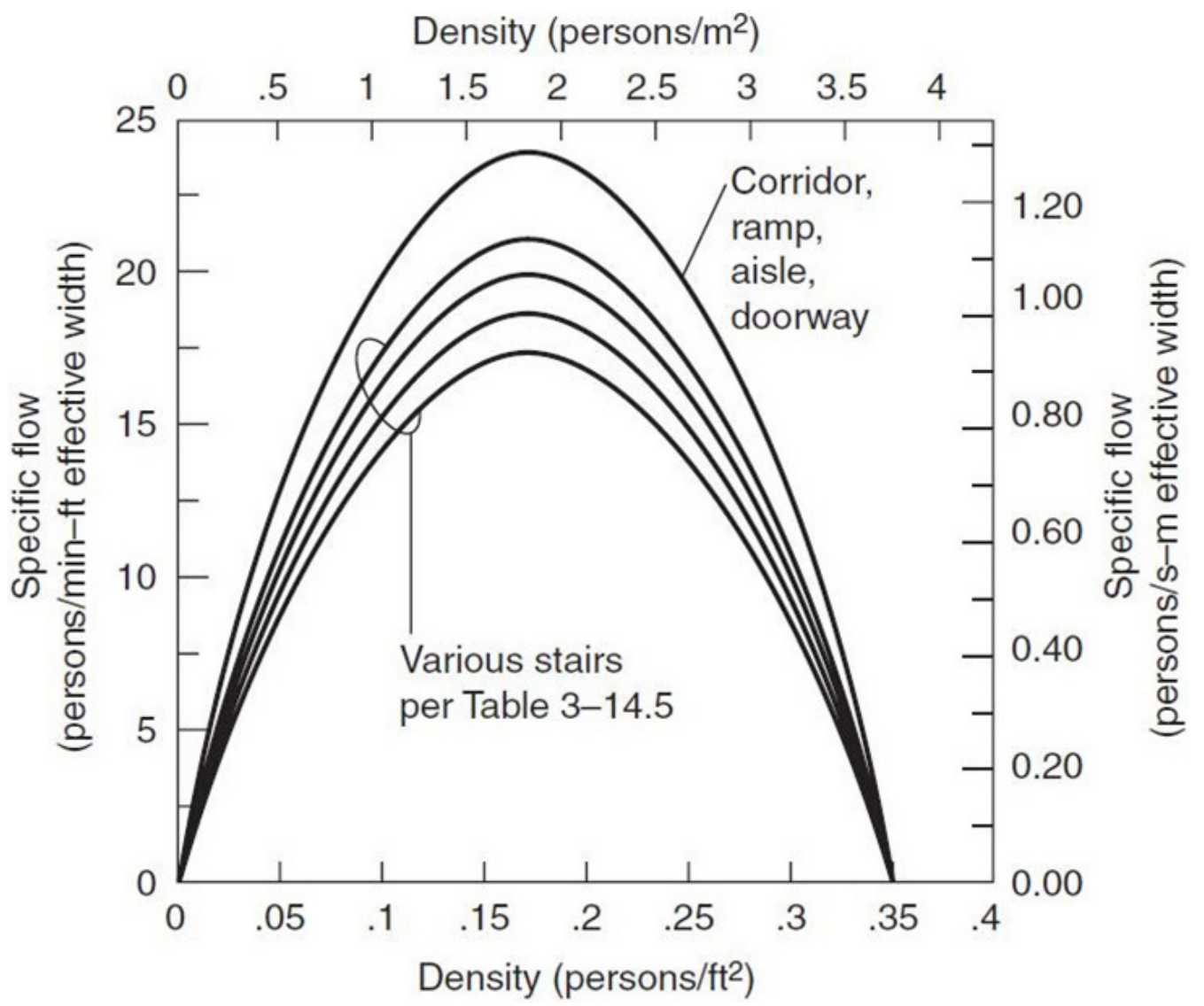

Figura 3. Flujo específico como función de la densidad. SFPE Handbook, Capítulo 13.

\subsection{Fases en la evacuación de las escaleras protegidas}

La primera consideración es que en el único análisis que puede hacerse, en base a los datos disponibles en el documento DB SI-3, es el tiempo de evacuación relacionado con el uso de la escalera, ya que no hay datos para valorar el tiempo que transcurre desde que los ocupantes comienzan a desplazarse hasta que llegan al acceso de la escalera ( $t$ tra (pres) en la terminología de la ISO). Esto significa que te $=0$, se correspondería con a un $\mathrm{t}>\mathrm{o}$.

Para evitar confusión con la terminología de la ISO, denominaremos al tiempo total de evacuación utilizando la escalera t paso y no t tra (queue). La razón para ello es que, como se verá, se producen cambios importantes en los flujos en los accesos a la escalera en las diferentes fases consideradas. Mientras en la fase de llenado el flujo está gobernado únicamente por la anchura de la escalera y es constante, en la fase posterior de vaciado los flujos dependen de la altura de la planta considerada y son variables en el tiempo. Así se evita la terminología "tiempo de cola" para abarcar condiciones muy diferentes. El tiempo total de evacuación utilizando la escalera será la suma del tiempo de conflencia (tconf) y el tiempo de espera (tesp) en las diferentes plantas

$$
\mathrm{t} \text { paso }=\mathrm{t} \operatorname{conf}+\mathrm{t} \text { esp }
$$

Las cinco fases se representan en la Fig. 4:

- Fase $1($ te $=0)$. El primer ocupante llega a la entrada de la escalera.
- Fase 2 (o < te < t conf). La escalera comienza a llenarse.

- Fase 3 (te = t conf). Se produce el llenado de todos los tramos de escalera y los flujos comienzan a mezclarse

- Fase 4 ( $\mathrm{t}$ conf $<$ te $<$ t paso). Los flujos se van mezclando en una proporción 50:50 (23). El Flujo de ocupantes que salen de la escalera en la planta de salida se equilibra con el sumatorio de los flujos de entrada en la escalera en las diferentes plantas. Los ocupantes, por tanto, esperan en los accesos (evitamos decir que hacen cola en ellos) en el t esp.

- Fase $5(\mathrm{t}=\mathrm{t}$ paso). El último ocupante entra en la escalera y se considera finalizada la evacuación

\subsection{Asunción para la estimación del ASET}

En el Documento Básico SI-3 no hay ninguna indicación que permita deducir el tiempo disponible para la evacuación en condiciones de seguridad más allá de la consideración, ya aludida, de que las fórmulas están establecidas bajo la hipótesis de que todos los ocupantes pueden traspasar una salida en un tiempo máximo de 2,5 minutos (150 segundos). Ese dato, sin embargo, es algo confuso, ya que se refiere solamente al paso de los ocupantes por unos elementos de evacuación concretos que, como se ha comentado, pueden formar parte de una serie.

Asumimos como único dato posible que el tiempo máximo disponible de espera en la escalera ( $t$ esp), que es el tiempo máximo que los ocupantes de las plantas pueden tardar en entrar en la escalera, tras el llenado de la misma, es 2,5 minutos. 
$t=0$ Los oapsintes están en la

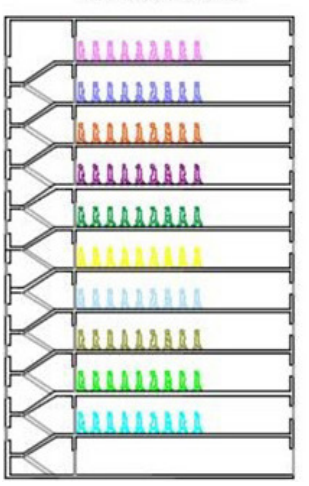

$0<t<$ tconf Se llena la escaler

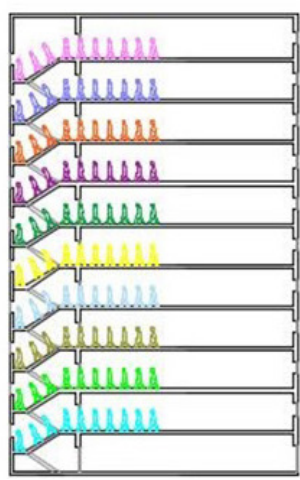

$t=t$ conf

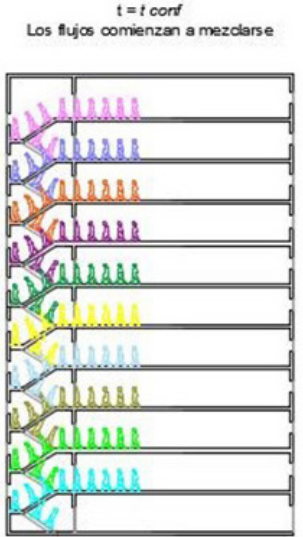

$t$ conf $<t<t$ paso

Se iguala el Flujo de salida de la escalera con la las Flujas de entrada en las plartas

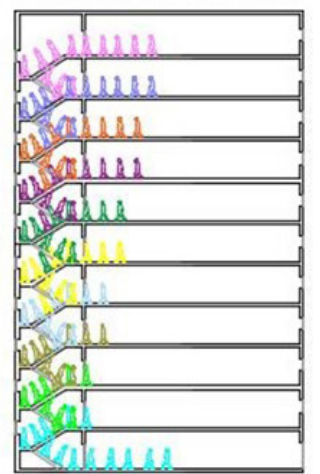

$\mathrm{t}=t_{\text {paso }}$

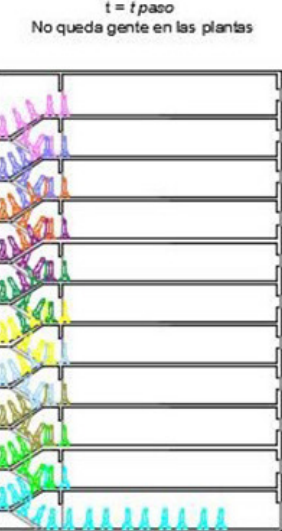

Figura 4. Fases de la evacuación vertical en escaleras protegidas de acuerdo con el CTE.

De acuerdo con ello, el tiempo máximo disponible para la evacuación sería la suma del tiempo de llenado o tiempo hasta la confluencia, t conf más 2,5 minutos.

\subsection{Asunción para la estimación del RSET}

De acuerdo con la hipótesis de que los ocupantes puedan hacer uso de una Salida de Planta en 2,5 minutos podemos considerar que los flujos considerados en el DB-SI3 son:

- $200 \mathrm{P} / \mathrm{m} / 150 \mathrm{~s}=1,33 \mathrm{P} / \mathrm{s} / \mathrm{m}$ para el movimiento en horizontal

- $160 \mathrm{P} / \mathrm{m} / 150 \mathrm{~s}=1,067 \mathrm{P} / \mathrm{s} / \mathrm{m}$ para el movimiento en las escaleras

Ambos Flujos Específicos, de acuerdo con la Fig. 4, pueden considerarse máximos, y se corresponden con densidades de $1,8 \mathrm{p} / \mathrm{m} 2$.

\subsection{Máxima capacidad de la escalera}

La capacidad máxima de la escalera es función directa de su superficie, al haber asumido el legislador una densidad de $3 \mathrm{p} / \mathrm{m} 2$

$$
\text { Pte }=\mathrm{n} \cdot 3 \cdot \mathrm{Se}
$$

\subsection{Tiempo de llenado de la escalera}

En un momento concreto (t conf), los ocupantes del piso "i“ llegan al piso "i-1". Esto se hace con la asunción de que t conf corresponde con el momento en el que la escalera se llena de ocupantes.

$$
\mathrm{t} \operatorname{conf}=3 \mathrm{Se} / 1,067 \cdot \mathrm{A}
$$

Por definición de salida de planta, la evacuación finaliza cuando todos los ocupantes de todas las plantas están dentro de la escalera en el momento t paso

$$
\mathrm{P}=\mathrm{n} \cdot 3 \cdot \mathrm{Se}+(\mathrm{t} \text { paso }-\mathrm{t} \text { conf }) \cdot 1.067 \cdot \mathrm{A}
$$

Cuando la escalera está llena de ocupantes, comienza la etapa en la que es necesario equilibrar el flujo de salida de la escalera en la planta de salida (160A), con los flujos de entrada de los ocupantes desde las diferentes plantas. En definitiva, se trata de que:

$$
\mathrm{Fe}=1,067=\sum \mathrm{Fi}
$$

Si el número de ocupantes de cada planta fuera infinito, la relación entre los flujos sería la que se presenta en la Fig. 5, ya que se considera una convergencia de flujos 50:50. Esto favorece los flujos procedentes de las plantas más bajas, que serían las que aportarían más ocupantes al flujo interior en las escaleras inicialmente. En este caso el modelo seguiría funcionando de forma continua.

$$
\mathrm{Fe}=\mathrm{Fe} / 2+\mathrm{Fe} / 4+\mathrm{Fe} / 8+\ldots .+2 \mathrm{Fe} / 2^{\wedge}(\mathrm{n}-1)
$$

Si el número de ocupantes de cada planta fuera finito, como ocurre en la realidad, el modelo comienza a tener importantes distorsiones, y habría que analizar su funcionamiento planta por planta. Como simplificación, para cálculos con no excesiva complejidad, se propone el siguiente procedimiento:

1. Comprobación de que en todas las plantas el número de ocupantes es mayor que el número capaz de albergar la escalera entre dos plantas consecutivas, 3Se.

2. Obtención de los restos de ocupantes que, por planta, no han entrado en la escalera en te $=t$ conf.

3. Obtención de los tiempos de espera ( $\mathrm{t}$ esp) en cada planta, que no deben superar los 150 segundos.
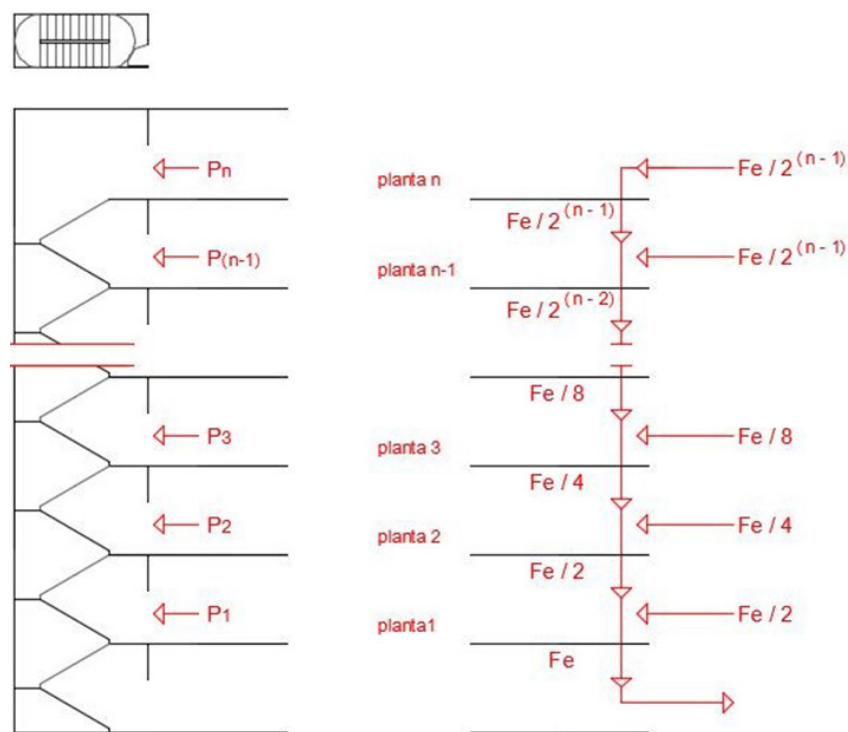

Figura 5. Flujos en escalera con ratio de convergencia de flujos 50:50. 
4. En el caso de que en alguna planta se exceda este tiempo, habría que calcular el número de personas que no hayan alcanzado la salida de planta y disponer de un área protegida previa.

\section{CASOS DE ESTUDIO: COMPARACIÓN CON SIMULACIÓN}

Se plantean dos casos de estudio de un mismo edificio con 8 plantas y una ocupación total de 520 ocupantes en ambos casos. El primero de ellos el caso extremo de un edificio con ocupaciones muy bajas en plantas bajas y alta ocupación en plantas elevadas

\section{Caso 1}

Resultados mostrados en la Tabla 5 .

\section{Caso 2}

\section{Resultados mostrados en Tabla 6.}

En los casos de las plantas 7 y 8, en los que se excede el tiempo de espera, y para no aumentar la anchura de la escalera, se puede diseñar un área de descompresión previa a la escalera. Como 78 personas por planta no han conseguido alcanzar el interior de la escalera en ese tiempo, su superficie sería de $78 / 3=26 \mathrm{~m} 2$

Se han realizado simulaciones con Pathfinder en este segundo caso, como se ve en la Figura 6, dado que este programa utiliza para el cálculo de la evacuación el modelo presentado por la SFPE en el Society of Fire Protection Handbook para el cálculo de movimiento de personas en las escaleras. El programa Pathfinder considerarándose para los ocupantes una velocidad de deplazamiento de $1,19 \mathrm{~m} / \mathrm{s}$, basado en lo expuesto en (22) y una anchura de hombros de $45,58 \mathrm{~cm}$ con una distancia de confort de $0.08 \mathrm{~m}$, ambos datos basados en los estudios de Fruin (24). De las simulaciones realizadas, sólo se recoge a continuación la más favorable: aquella en la que no se considera a ningún ocupante con problemas de movilidad ni tiempo de reacción, de manera que se inicia la evacuación de forma instantánea. Esto implica que otras consideraciones más pesimistas conllevarían mayor tiempo para la evacuación que el recogido en el ejemplo.

Se han obtenido los siguientes valores:

- $\mathrm{t} \operatorname{conf}=38 \mathrm{~s}$

- t paso de $400 \mathrm{~s}$ en planta $7^{\mathrm{a}}$ y $440 \mathrm{~s}$ en planta $8^{\mathrm{a}}$. Estos darían t esp de 362 s y 402 s respectivamente

Tabla 5. Caso 1.

\begin{tabular}{|c|c|c|c|c|c|c|c|}
\hline $\begin{array}{c}\text { Número de } \\
\text { planta }\end{array}$ & $\mathbf{P i}$ & $\begin{array}{c}\text { Características } \\
\text { Escalera }\end{array}$ & $\begin{array}{l}\text { Resto } P \\
\text { en tconf }\end{array}$ & $\begin{array}{c}\text { Fi } \\
\text { inicial }\end{array}$ & $\frac{\operatorname{tesp}(*)}{s}$ & Adec. & $\begin{array}{c}\text { Exceso } \\
\text { de tiempo }\end{array}$ \\
\hline 8 & 65 & \multirow{10}{*}{$\begin{array}{c}A=1,2 \mathrm{~m} \\
\mathrm{Pe}=41 \mathrm{p} \\
\mathrm{Se}=13,67 \mathrm{m2} \\
\text { t } \operatorname{conf}=32,029\end{array}$} & 24 & $\mathbf{0 , 0 0 8}$ & 150 & Sí & - \\
\hline 7 & 65 & & 24 & $\mathbf{0 , 0 0 8}$ & 150 & Sí & - \\
\hline 6 & 65 & & 24 & 0.016 & 131,32 & Sí & - \\
\hline 5 & 65 & & 24 & $\mathbf{0 , 0 3 3}$ & 112,56 & Sí & - \\
\hline 4 & 65 & & 24 & 0,066 & 93,8 & Sí & - \\
\hline 3 & 65 & & 24 & 0,133 & 75,04 & Sí & - \\
\hline 2 & 65 & & 24 & 0,266 & 56,28 & Sí & - \\
\hline $\mathbf{1}$ & 65 & & 24 & $\mathbf{0 , 5 3 3}$ & 37,52 & Sí & - \\
\hline B & & & & & & & \\
\hline & $\begin{array}{l}\text { Total } \\
520 \text { p }\end{array}$ & & & $\begin{array}{l}\sum \mathbf{F i}= \\
\mathbf{1 , 0 6 7}\end{array}$ & & & \\
\hline
\end{tabular}

t esp admisible $=\mathbf{1 5 0} \mathbf{~} \mathbf{s}$

$t$ esp $1=24 / 0,533 \times 1,2=\mathbf{3 7 , 5 2} \mathbf{~ s}$

$\left(^{*}\right)$ tesp $i=t \operatorname{esp} 1(1+(n-1) / 2)$

Tabla 6. Caso 2.

\begin{tabular}{|c|c|c|c|c|c|c|c|}
\hline $\begin{array}{l}\text { Número de } \\
\text { planta }\end{array}$ & $\mathbf{P i}$ & $\begin{array}{c}\text { Características } \\
\text { Escalera }\end{array}$ & $\begin{array}{l}\text { Resto } P \\
\text { en tconf }\end{array}$ & $\begin{array}{c}\text { Fi } \\
\text { inicial }\end{array}$ & $\underset{s}{t \operatorname{esp}\left({ }^{*}\right)}$ & Adec. & $\begin{array}{c}\text { Exceso } \\
\text { de tiempo }\end{array}$ \\
\hline 8 & 215 & \multirow{10}{*}{$\begin{array}{c}A=1,2 \mathrm{~m} \\
\mathrm{Pe}=41 \mathrm{p} \\
\mathrm{Se}=13,67 \mathrm{m2} \\
\text { t } \operatorname{conf}=32,029\end{array}$} & 174 & o,533 & 272,04 & No & 122,04 \\
\hline 7 & 215 & & 174 & o,533 & 272,04 & No & 122,04 \\
\hline 6 & 15 & & - & - & No & Sí & \\
\hline 5 & 15 & & - & - & No & Sí & \\
\hline 4 & 15 & & - & - & No & Sí & \\
\hline 3 & 15 & & - & - & No & Sí & \\
\hline 2 & 15 & & - & - & No & Sí & \\
\hline 1 & 15 & & - & - & No & Sí & \\
\hline B & & & & & & & \\
\hline & $\begin{array}{l}\text { Total } \\
520 \text { p }\end{array}$ & & & $\begin{array}{l}\sum \mathbf{F i}= \\
\mathbf{1 , 0 6 7}\end{array}$ & & & \\
\hline
\end{tabular}

t esp admisible $=\mathbf{1 5 0} \mathbf{~}$

t esp $1=174 / 0,533 \times 1,2=\mathbf{2 7 2 , 0 4}$ s

$\left.{ }^{*}\right)$ t esp $i=t$ esp $1(1+(n-1) / 2)$

150 s $x 0.533 \mathrm{p} / \mathrm{s} / \mathrm{m} \times 1.2 \mathrm{~m}=96 \mathrm{p}$. Por tanto, en $150 \mathrm{~s}$ ( $\mathrm{t}$ esp) pueden evacuar 96 personas, si había 174 personas en $t$ conf, quedan sin llegar al interior de la escalera $78 \mathrm{p}$. 

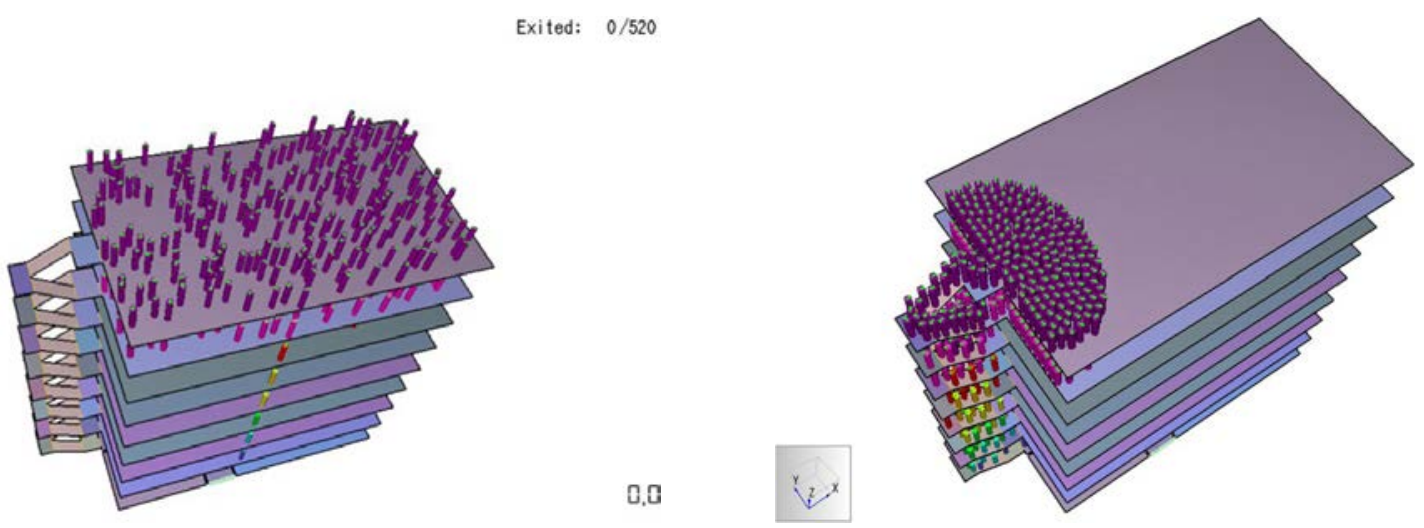

Figura 6. Imágenes de simulación en planta $8^{\mathrm{a}}$ : te $=$ o y te $=\mathrm{t}$ conf.

La diferencia de t conf entre los cálculos manuales y la simulación se deben fundamentalmente a que en ningún momento se alcanzan los 41 ocupantes entre dos tramos de escalera, sino un máximo de 37. Este dato alerta sobre la alta densidad considerada por el CTE en el interior de las escaleras, que en la literatura reciente se limita a 2,10 p/m2 (25).

Las diferencias de t paso, del orden de un 40\% de media superiores en la simulación, se pueden deber al hecho de que el CTE no incluye los tiempos de desplazamiento de los ocupantes desde los orígenes de evacuación hasta la puerta de la escalera, haciendo muy difícil representar la situación ideal considerada en te $=0$. En cualquier caso las diferencias son suficientemente significativas para parecer preocupantes.

\section{DISCUSIÓN}

Los cálculos manuales y las simulaciones realizadas, reflejan que, bajo determinadas condiciones de ocupación, los tiempos de paso y espera en el acceso a las escaleras protegidas exceden ampliamente los considerados en la legislación española. Este puede ser el caso de locales de pública concurrencia en plantas altas de edificios de cierta altura.

El análisis realizado en este artículo no ha tenido en cuenta, además, importantes condicionantes que agravarían seriamente el problema, como es la consideración de personas con problemas de movilidad (26)(27).

Se intuye de forma clara que el mismo problema, en mayor medida si cabe, existe en el cálculo de los pasillos protegidos.

\section{CONCLUSIONES}

- El documento DB SI-3 presenta métodos para el cálculo de los elementos de evacuación de edificios puramente prescriptivos. A partir de su revisión, mediante un análisis inverso, es posible alumbrar algunos de los parámetros asumidos por el legislador, que se encuentran tras la apariencia mágica de las experesiones propuestas. Algunos de esos parámetros, como el flujo de evacuación, se corresponden con valores considerados óptimos en la literatura especializada. Otros pueden ser contradictorios, como la excesiva densidad considerada en las escaleras para que el flujo sea el deseado.

- El modelo elegido para predecir el movimiento de los ocupantes en las escaleras protegidas en caso de incendio muestra su debilidad en edificios que tienen plantas con ocupaciones muy diferentes, fundamentalmente si las de mayor ocupación están en las plantas más altas. Aparecen zonas desocupadas en el interior de la escalera, mientras los ocupantes se agolpan ante algunas salidas, excediendo claramente el tiempo considerado.

- La coordinación de los flujos entre los accesos en todas las plantas a las escaleras protegidas y el flujo en su interior y en la planta de desembarco es crucial. Para garantizarlo se pueden utilizar estrategias de diseño, como las áreas de descompresión previas en accesos complicados.

- El diseño y cálculo de las escaleras protegidas, debería incluir una caracterización del edificio y de sus ocupantes. El diseño prestacional constituye la única aproximación posible en el caso de edificios complejos.

\section{REFERENCIAS}

(1) Gissi, E., Ronchi, E., Purser, D. A. (2017). Transparecy vs magic numbers: The development of stair design requirements in the Italian Fire Safety Code. Fire Safety Journal, doi: http://dx.doi.org/10.1016/j.firesaf.2017.03.037

(2) Gobierno de España (2006). Real Decreto 314/2006 por el que se aprueba el Código Técnico de la Edificación. Boletín Oficial del Estado $n^{0} 74$. España.

(3) Meacham, Brian J. (2004). Performance-Based Building Regulatory Systems: Structure, Hierarchy and Linkages. Journal of the Structural Engineering Society of New Zealand, 17 (1): 37-51

(4) Meacham, B. J., Moore, A., Bowen, R., Traw, J. (2005). Performance-Based Building Regulation: Current Situation and Future Needs. Building Research \& Information. (33, 1): 91-106

(5) European Parliament (2011). Regulation (EU) No $305 / 2011$ laying down harmonised conditions for the marketing of construction products and repealing Council Directive 89/106/EEC. Official Journal of the European Union. OJ L 88, 4.4.2011. EU.

(6) Gobierno de España (1981). Real Decreto 2059/1981 por el que se aprueba la Norma Básica de la Edificación «Condiciones de protección contra incendio en los edificios». Boletín Oficial del Estado nº 224. España. 
(7) Gobierno de España (1991). Real Decreto 279/1991 por el que se aprueba la Norma Básica de la Edificación «NBE: CPI/91: Condiciones de protección contra incendio en los edificios». Boletín Oficial del Estado n ${ }^{0}$ 58. España.

(8) Gobierno de España (1996). Real Decreto 2177/1996 por el que se aprueba la Norma Básica de la Edificación «NBE: CPI/96: Condiciones de protección contra incendio en los edificios». Boletín Oficial del Estado n ${ }^{261}$. España.

(9) Gobierno de España (2006). Real Decreto 314/2006 por el que se aprueba el Código Técnico de la Edificación. Documento Básico SI, Seguridad en caso de incendio. Boletín Oficial del Estado n⿳0 74 . España.

(10) Blair, A. J., Milke, J. A. (2011). The Effect of Stair Width on Occupant Speed and Flow Rate for Egress of High Rise Buildings. En Peacock, R. D., Kuligowski, E. D, Averill, J. D. (Eds.), Pedestrian and Evacuation Dynamics. (pp. 747-750)

(11) Peacock, R. D., Averill, J. D., Kuligowski, E. D. (2009). Stairwell Evacuation from Buildings: What We Know We Don't Know. NIST Technical Note 1624 (pp. 1-16)

(12) The Ministry of Works (1952). Fire Grading of Buildings, Part II. Fire Fighting Equipment, Part III. Personal safety, Part IV. Chimneys and flues. Her Majesty's Stationery Office, Post-War Building Studies, No. 29. Great Britain.

(13) Ross, L. (2012). Invitation and Escape: The Architecture of fire Safety Regulation. Theory by design. Antwerp. https:// docs.google.com/file/d/oB2IoXCL77YSMQk11NDBBS3JlaXM/edit?pli=1

(14) Rey, E., Aguayo, F., Peralta, M. E., Lama, J. R., Ávila M. J. (2015). Integración de métodos escalares y vectoriales en la evaluación del riesgo de incendio en el ciclo de vida de una construcción. Informes de la Construcción, 67 (539): 1-11

(15) ISO (2009). ISO/TR 16738:2009 Fire-safety engineering- Technical information on methods for evaluating behaviour and movement of people. International Standards Organization.

(16) Faller, G. (2003). Seguridad contra incendios en edificios con atrio. Informes de la Construcción, 55 (485), pp. 27-32

(17) Nelson, H. E., Mowrer, F.W. (2002). Emergency Movement. En SFPE Handbook of Fire Protection Engineering (3rd ed.)(367-380). Quincy, MA: National Fire Protection Engineering

(18) Fruin, J. (1971). Pedestrian Planning and Design. New York: Metropolitan Association of Urban Designers and Environmental Planners

(19) Fruin, J. (1987). Pedestrian Planning and Design (ed. rev.). Mobile, AL: Elevator World

(20) Pauls, J. L. (1977). Management of and Movement of Building Occupants in Emergencies. (DBR Paper No 788). Ottawa: National Research Council Canada

(21) Predtechenskii, V. M., Milinskii, A. I. (1978). Planning for Foot Traffic Flow in Buildings. New Delhi: Amerind

(22) Proulx, G. (2002). Movement of People. En SFPE Handbook of Fire Protection Engineering (3rd ed.) (342-366). Quincy, MA: National Fire Protection Engineering

(23) Sano, T., Ronchi, E., Minegishi, Y., Nilsson, D. (2017). A pedestrian merging flow model for stair evacuation. Fire Safety Journal, 89: 77-89

(24) Fruin, J. J. (1987) Pedestrian Planning and Design, (Revised Edition). Elevator World, Inc, Mobile, AL.

(25) Hostikka, S., Paloposki, T., Rinne, T., Saari, J. M., Korhonen, T., Heliövaara, S. (2007). Evacuation Experiments in Offices and Public Buildings. VTT Technical Research Center of Finland

(26) Kuligowski, E. D., Peacock, R. D., Wiess, E., Hoskins, B. (2013). Stair evacuation of older adults and people with mobility impairments. Fire Safety Journal, 62: 230-237

(27) Boyce, K. E., Shields, T. J., Silcock G. W. H. (1999). Toward the characterization of building occupancies for Fire Safety Engineering: Prevalence, type, and mobility of disabled people. Fire Technology, 35(1): 35-50. 